\title{
Comparison of Average Energy Slope Estimation Formulas for One-dimensional Steady Gradually Varied Flow
}

\author{
Wojciech Artichowicz, Patrycja Mikos-Studnicka
}

Gdańsk University of Technology, Faculty of Civil and Environmental Engineering, ul. G. Narutowicza 11/12, 80-233 Gdańsk, Poland, e-mail: wojciech.artichowicz@wilis.pg.gda.pl

(Received October 09, 2014; revised December 03, 2014)

\begin{abstract}
To find the steady flow water surface profile, it is possible to use Bernoulli's equation, which is a discrete form of the differential energy equation. Such an approach requires the average energy slope between cross-sections to be estimated. In the literature, many methods are proposed for estimating the average energy slope in this case, such as the arithmetic mean, resulting in the standard step method, the harmonic mean and the geometric mean. Also hydraulic averaging by means of conveyance is commonly used. In this study, water surface profiles numerically computed using different formulas for expressing the average slope were compared with exact analytical solutions of the differential energy equation. Maximum relative and mean square errors between numerical and analytical solutions were used as measures of the quality of numerical models. Experiments showed that all methods gave solutions useful for practical engineering purposes. For every method, the numerical solution was very close to the analytical one. However, from the numerical viewpoint, the differences between the methods were significant, as the errors differed up to two orders of magnitude.
\end{abstract}

Key words: open channel, steady flow, gradually varied flow, standard step method, energy slope

\section{Introduction}

Steady gradually varied flow (SGVF) is one of the main interests of practical open-channel hydraulics. Parameters of such flow are steady in time, but can vary in space because of changes in channel geometry, bed slope or the existence of lateral inflow or hydraulic structures. The method most frequently used for computations of one-dimensional SGVF is based on the discrete energy equation (Chanson 2004, Chow 1959, French 1985). This method results from Bernoulli's equation written for a reach of an open channel. For a channel reach bounded by the neighbouring cross-sections indexed $i$ and $i+1$ (Fig. 1), Bernoulli's equation has the following form:

$$
h_{i+1}+\alpha_{i+1} \frac{U_{i+1}^{2}}{2 g}=h_{i}+\alpha_{i} \frac{U_{i}^{2}}{2 g}-\Delta x_{i} \cdot \overline{S_{i}}
$$


where:

$\alpha-$ energy correctional coefficient,

$g$ - gravitational acceleration,

$h$ - water level elevation above the assumed datum,

$\bar{S} \quad-$ average slope of the energy line over the channel reach bounded by the cross-sections $i$ and $i+1$,

$U$ - average velocity in a cross section,

$\Delta x-$ step size (distance between the cross sections $i$ and $i+1$ ).

A graphical interpretation of Eq. (1) is displayed in Fig. 1.

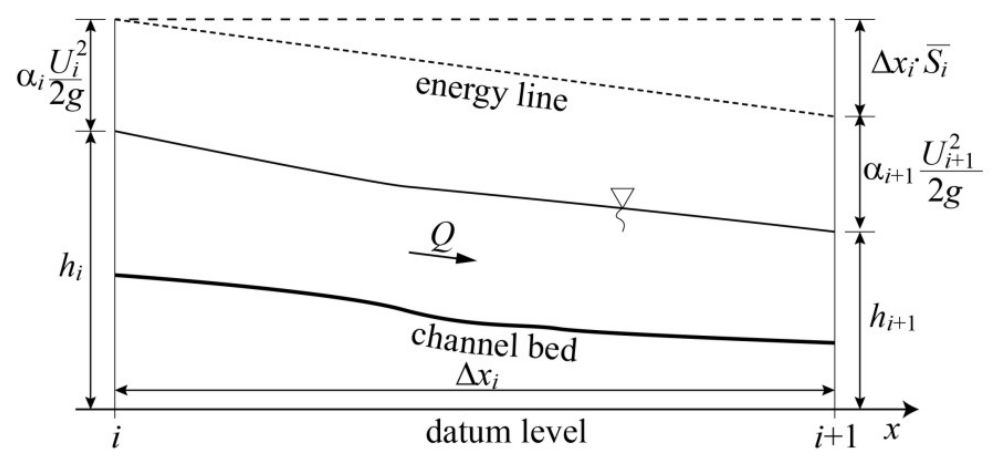

Fig. 1. Graphical interpretation of Eq. (1)

As all variables in Eq. (1) are related to the cross sections, the average slope of energy can be expressed by using only the nodal values. If the average energy slope $\overline{S_{i}}$ is estimated by the arithmetic mean

$$
\overline{S_{i}}=\frac{1}{2}\left(S_{i}+S_{i+1}\right),
$$

then the formula (1) becomes the well-known standard step method (Chanson 2004, Chow 1959, French 1985). The friction slope in a cross-section can be computed with Manning's formula:

$$
S_{i}=\frac{Q^{2} \cdot n^{2}}{R_{i}^{4 / 3} \cdot A_{i}^{2}},
$$

where $Q$ is a flow rate, $A$ is a wetted cross-sectional area, $R$ denotes a hydraulic radius, and $n$ is the Manning roughness coefficient, or with the Darcy-Weisbach formula (Chanson 2004):

$$
S_{i}=\frac{Q^{2} \cdot \lambda}{8 \cdot g \cdot R_{i} \cdot A_{i}^{2}}
$$

where $\lambda$ is a friction factor. 
In the literature regarding the subject of open-channel flow hydraulics, other formulas than the one given by Eq. (2) are proposed for the estimation of the average energy slope in the reach considered. For instance, the HEC-RAS software (US Army Corps of Engineers (2010)) uses the hydraulic mean, geometric mean and harmonic mean for the estimation of $\overline{S_{i}}$. The hydraulic mean of energy slopes is given by

$$
\overline{S_{i}}=\left(\frac{Q_{i}+Q_{i+1}}{K_{i}+K_{i+1}}\right)^{2},
$$

in which $K$ denotes conveyance:

$$
K_{i}=\frac{A_{i} \cdot R_{i}^{2 / 3}}{n} .
$$

If $Q=$ const, then Eq. (5) simplifies to

$$
\overline{S_{i}}=\left(\frac{2 Q}{K_{i}+K_{i+1}}\right)^{2} .
$$

Eq. (5) is a default averaging method in the HEC-RAS software. Geometric and harmonic mean averaging result in the following formulas, respectively:

$$
\begin{aligned}
\overline{S_{i}} & =\sqrt{S_{i} \cdot S_{i+1}}, \\
\overline{S_{i}} & =\frac{2 \cdot S_{i} \cdot S_{i+1}}{S_{i}+S_{i+1}} .
\end{aligned}
$$

When investigating the differences between the formulas given by Eqs. (2), (5), (6), (7) and (8), Laurenson (1986) showed that these formulas "systematically give different estimates" of the average energy slope. He also concluded that the arithmetic mean slope (Eq. (2)) is the safest and generally the best method. However, in some cases, it gives a slightly greater error of average slope estimation than the formulas given by Eqs. (5) to (8). Moreover, Chadderton and Miller (1980), when investigating the abovementioned slope averaging formulas, examined the relative magnitude of $\overline{S_{i}}$ based on the values of the friction slopes $S_{i}$ and $S_{i+1}$. They showed that the arithmetic mean slope gives systematically the highest values of the average slope, whereas the harmonic mean gives the lowest values. They also pointed out that all other formulas for slope estimation (e.g. those proposed by Reed and Wolfkill (1976)) give average slope values in between the values obtained by arithmetic and harmonic mean averaging.

The original research of Chadderton and Miller (1980) focused on finding the average slope estimation method that would return values lower than the harmonic mean. That solution would fit computations of the drawdown water stage profile. They proposed an improvement in average slope estimation for the drawdown water stage profile. 
A general formula proposed by Chadderton and Miller (1980) for average slope estimation is described by

$$
\overline{S_{i}}=C_{1} \cdot S_{i}+C_{2} \cdot S_{i+1},
$$

where the weighting parameters $C_{1}$ and $C_{2}$ are positive constants such that $C_{1}+C_{2}=$ 1. To find $C_{1}$ and $C_{2}$, the energy slope function $S(x)$ was assumed. For a drawdown curve, the authors used both parabolic and elliptic functions. The slope function is then integrated analytically at a distance from $x_{i}$ to $x_{i+1}$, giving values of $C_{1}$ and $C_{2}$ as a result. Finally, Chadderton and Miller obtained two formulas for the assumed parabolic $S(x)$ function:

$$
\overline{S_{i}}=\frac{1}{3} S_{i}+\frac{2}{3} S_{i+1}
$$

and for the elliptic one:

$$
\overline{S_{i}}=\left(1-\frac{\pi}{4}\right) \cdot S_{i}+\frac{\pi}{4} S_{i+1}
$$

There are also other types of averaging, in which formulas for the average slope are expressed by averaging the hydraulic radius, wetted perimeter and active flow areas, similarly as in Eq. (5). So the average energy slope is expressed by the mean values of hydraulic parameters rather than by manipulating the slope frictions $S_{i}$ and $S_{i+1}$ in cross-sections considered. Examples of such formulas can be found in French (1979) who quotes them from the original study by Reed and Wolfkill (1976)

$$
\begin{gathered}
\bar{S}=\frac{Q^{2} \cdot n^{2}}{\left[2 \cdot \frac{A_{i} \cdot A_{i+1}}{A_{i}+A_{i+1}}\right]^{2} \cdot\left[\frac{R_{i}+R_{i+1}}{2}\right]^{4 / 3}}, \\
\bar{S}=\frac{Q^{2} \cdot n^{2}}{\left[\frac{A_{i}+A_{i+1}}{2}\right]^{2} \cdot\left[\frac{A_{i}+A_{i+1}}{P_{i}+P_{i+1}}\right]^{4 / 3}}, \\
\bar{S}=\frac{Q^{2} \cdot n^{2}}{\left[\frac{A_{i}+A_{i+1}}{2}\right]^{2} \cdot\left[\frac{R_{i}+R_{i+1}}{2}\right]^{4 / 3}}, \\
\bar{S}=\frac{Q^{2} \cdot n^{2}}{\left[\frac{A_{i} \cdot R_{i}^{2 / 3}+A_{i+1} \cdot R_{i+1}^{2 / 3}}{2}\right]^{2}} .
\end{gathered}
$$

where $P$ denotes the hydraulic perimeter. It can be noticed that, in fact, Eq. (15) is identical to Eq. (6).

When considering SGVF, one can assume another viewpoint. The problem of finding the water stage profile when flow discharge is known, can be regarded as 
a problem of the numerical solution of an ordinary differential equation (ODE). Thus, the problem of averaging the energy slope can be seen as closely related to using different methods for the numerical solution of the energy ODE. On the other hand, for specific flow cases it is possible to obtain an analytical solution of the ODE for SGVF (MacDonald et al 1997). This fact makes it possible to compare the numerical results with the analytical solution of the SGVF ODE.

Reed and Wolfkill (1976) compared the results of slope averaging formulas with the results obtained by the numerical integration of the energy slope with Simpson's rule. Laurenson (1986) compared averaging formulas to the friction slope line assumed to be third-degree polynomial. Chadderton and Miller (1980) used HEC-2 numerical solutions as reference. To the best of the authors' knowledge, a comparison of numerical solutions obtained with different energy slope averaging formulas with analytic solutions of the SGVF equation has not been performed.

In further sections, the formulas given by Eqs (2), (5)-(15) for average energy slope estimation will be used in computations of water profiles. The results of numerical experiments will be compared with analytical benchmark solutions, three of which represent tranquil flow, and one represents rapid flow.

\section{Governing Equation}

The governing equation of SGVF can be derived in many ways (Szymkiewicz 2010). Using the energy principle, the following one-dimensional equation can be derived:

$$
\frac{d}{d x}\left(h+\frac{\alpha \cdot Q^{2}}{2 g \cdot A^{2}}\right)=-S .
$$

In an alternative form, Eq. (16) can be expressed as

$$
\frac{d E}{d x}=-S
$$

with

$$
E=h+\frac{\alpha \cdot Q^{2}}{2 g \cdot A^{2}} .
$$

Equation (17) is an ODE. It should be noted that there are other possible forms of the governing equation of SGVF. If the flow rate $Q$ is known, then the initial-value problem (IVP) for Eq. (17) can be stated (Ascher and Petzold 1998, Kincaid and Cheney 2006, Szymkiewicz 2010). The IVP requires that the energy stage at one of the bounds of the solution domain (i.e. channel reach) is given (Szymkiewicz 2010). If flow is tranquil, then the energy stage should be imposed in the outflow cross-section, so the initial condition will take the following form (Fig. 2a):

$$
E_{L}=E\left(x_{L}=L\right)
$$



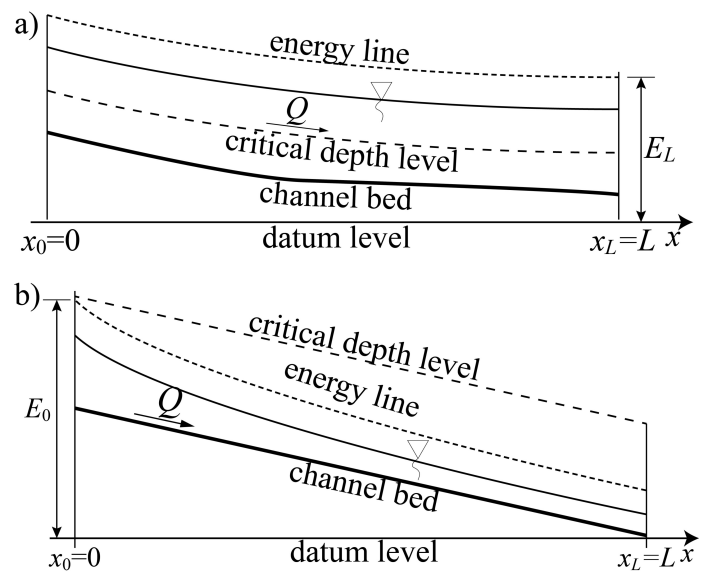

Fig. 2. Graphical representation of the channel and required initial values

with $L$ denoting the length of the channel. In the case of rapid flow, the energy stage must be imposed in the inflow cross-section, and the initial value becomes (Fig. 2b):

$$
E_{0}=E\left(x_{0}=0\right) \text {. }
$$

In practice, this means that the solution of the IVP for Eq. (17), requires water stage in the first or last cross-section of the channel to be given. In this way, the IVP can be formulated and solved even when the water flow profile passes through the critical depth (Artichowicz and Szymkiewicz 2014).

Consider the IVP for a general ODE:

$$
\frac{d y(x)}{d x}=f(x, y(x))
$$

with

$$
y_{0}=y\left(x=x_{0}\right),
$$

where:

$$
\begin{array}{ll}
x & - \text { independent variable, } \\
y(x) & - \text { dependent variable, } \\
f(x, y)=y^{\prime}(x) & - \text { derivative of the function } y(x), \\
y_{0}=y\left(x=x_{0}\right) & - \text { initial value. }
\end{array}
$$

If the problem stated by Eqs. (21) and (22) cannot be solved analytically, it is necessary to apply numerical methods. Detailed descriptions of applicable methods, their advantages and disadvantages can be found, for example, in Ascher and Petzold (1998), Hairer et al (2005), Kincaid and Cheney (2002) and many other publications. However, for open-channel flow, the most suitable methods are those which involve the flow and channel parameters from the measured cross-sections, i.e. those which do 
not require interpolation between cross-sections. Applications of such methods to hydraulic engineering problems are presented, for example, by Cunge et al (1979) and Szymkiewicz (2010).

The approach developed by Chadderton and Miller (1980) is, in fact, an application of the so-called general two-level formula for numerical integration of ODEs to the energy equation. It is a generalization of one-step numerical methods used for the numerical solving of ODEs. The general two-level formula is expressed as follows:

$$
y_{i+1}=y_{i}+\Delta x_{i} \cdot\left((1-\theta) \cdot f\left(x_{i}, y_{i}\right)+\theta \cdot f\left(x_{i+1}, y_{i+1}\right)\right),
$$

in which $\theta \in[0,1]$ is a weighting parameter that makes it possible to control the order of the approximation scheme. For $\theta=0$, Eq. (23) becomes the explicit (forward) Euler formula, for $\theta=1$, the implicit (backward) Euler method, and for $\theta=1 / 2$, the implicit trapezoidal rule. If $\theta=2 / 3$, then Eq. (23) becomes the formula proposed by Chadderton and Miller (1980), corresponding to the one commonly known as Galerkin's formula.

Applying formula (23) to energy equation (17), one obtains:

$$
E_{i+1}=E_{i}+\frac{\Delta x_{i}}{2}\left(-(1-\theta) \cdot S_{i}-\theta \cdot S_{i+1}\right) .
$$

The introduction of the average flow velocity $U=Q / A$ and Eq. (18) yields:

$$
h_{i+1}+\alpha_{i+1} \frac{U_{i+1}^{2}}{2 g}=h_{i}+\alpha_{i} \frac{U_{i}^{2}}{2 g}-\frac{\Delta x_{i}}{2}\left((1-\theta) \cdot S_{i}+\theta \cdot S_{i+1}\right) .
$$

One of the most popular methods in open-channel hydraulics is the implicit trapezoidal rule. It has very good numerical properties fitting the needs of open-channel hydraulics (Szymkiewicz 2010). When the trapezoidal rule is applied $(\theta=1 / 2)$, then Eq. (25) becomes:

$$
h_{i+1}+\alpha_{i+1} \frac{U_{i+1}^{2}}{2 g}=h_{i}+\alpha_{i} \frac{U_{i}^{2}}{2 g}-\frac{\Delta x_{i}}{2}\left(S_{i}+S_{i+1}\right) .
$$

Note that Eq. (26) is the standard step method given by formula (1) with Eq. (2) (Chow 1959, Cunge et al 1979, French 1985, Szymkiewicz 2010, US Army Corps of Engineers 2010). Therefore, the approximation of energy equation (17) with the trapezoidal rule leads to average energy slope estimation by the arithmetic mean. Note that other numerical methods of solving ODEs imply other formulas for averaging the slope energy. Therefore, the application of the previously listed methods means that different methods for solving the IVP for ODE (17) are used. However, this question is beyond the scope of this paper.

Eqs (25) and (26) are algebraic non-linear equations with only one unknown. All other variables are known from the initial condition or from the previous step of computation. By solving this equation in subsequent cross-sections iteratively, it is possible to find the water stage profile along the channel. 


\section{Benchmark Solution Test Cases}

The best way to test the quality of the numerical methods of solving ODEs is to compare the outcome of computations with the analytic solution. Therefore, the analytic benchmark solution methodology described by MacDonald et al (1997) was used. Although MacDonald et al (1997) derived their methodology for the SGVF equation in the form proposed by Chow (1959), it was possible to apply it to the energy equation in the form of Eq. (17) as is. This approach relies on the construction of the inverse problem for the SGVF equation, in which the bed level function $z(x)$ is estimated for a given or assumed depth function $H(x)$. To find $z(x)$, one also needs to know cross-sectional geometry: the channel width $B(x, H(x))$, active flow area $A(x, H(x))$, and wetted perimeter $P(x, H(x))$, as well as the function expressing the friction slope $S(x, H(x))$. The bed slope function is given by

$$
s_{b}(x)=\frac{d H}{d x}+S(x, H(x))+\frac{\alpha \cdot Q^{2}}{2 g} \frac{d}{d x}\left(\frac{1}{A^{2}(x, H(x))}\right) .
$$

The bed level corresponding to the imposed depth function $H(x)$ and channel geometry can be found by integrating the bed slope function:

$$
z(x)=-\int_{x}^{L} s_{b}(\chi) d \chi .
$$

If the analytical integration of $z(x)$ is impossible, then high accuracy numerical quadrature methods can be used, which give nearly exact result. Therefore, the analytic functions $H(x)$ and $S(x, H(x))$ can be used as perfect reference for testing the SGVF numerical solvers.

Computations of water stage in the test cases were performed by means of Bernoulli's equation (Eq. (1)) with different mean slope averaging formulas applied:

- arithmetic mean (Eq. (2));

- hydraulic averaging with Eq. (6);

- geometric mean (Eq. (7));

- harmonic mean (Eq. (8));

- averaging with coefficients obtained by assuming a parabolic function (Eq. (10));

- averaging with coefficients obtained by assuming an elliptic function (Eq. (11));

- Eqs 12-15 denoted in figures and tables by the initials of their authors as RW1, RW2, RW3 and RW4 respectively.

In all test cases channels were assumed to have rectangular cross-sections. Also in each case, the energy slope function expressed with Manning's formula (Eq. (3)) with a constant roughness coefficient was used.

Four test cases typical of open-channel hydraulics were considered. The test cases were selected to determine whether formulas recommended for specific water profile 
types would prove to be the best compared with analytical solutions. Examples were also selected in such a way that the shapes of energy slope functions would be significantly different. The first test case represents a channel with a variable bed slope. In this case, the energy slope function has inflection points, as well as local maxima and minima, so it can represent river flow profiles in which the channel bed slope varies along the river. The second test case represents a drawdown water flow profile for which the energy slope is a function increasing exponentially along with the flow direction. Such a profile is typical of channels with a pending outflow cross-section or of channels with a bed slope changing from subcritical to supercritical. Formulas given by Eqs (10) and (11) examined in this paper were derived especially for such a water profile. Test case 3 is a backwater curve commonly found in open channels crossed with a dam or an overflow. The last test case represents rapid flow in a spillway with an increasing bed slope. The energy slope is an increasing one, and it reaches much higher values than in the previous test cases.

In each test case, the numerical solution for each slope averaging method was compared with the analytic solution. The comparison was made for the depth function $H_{i}$, obtained by computation, and the analytic depth function $H(x)$, estimated in computational cross-sections $x_{i}$, that is $H\left(x_{i}\right)$.

Computations were conducted with two step sizes $\Delta x$ for each averaging method: the first step size used was ten times as large as the second. This made it possible to estimate error reduction when decreasing the step length by $90 \%$. Errors examined were

- the maximum relative error:

$$
\delta_{\max }=\max \left(\frac{\left|H_{i}-H\left(x_{i}\right)\right|}{H\left(x_{i}\right)}\right) ;
$$

- the mean square error:

$$
M S E=\frac{1}{N} \sum_{i}\left(H_{i}-H\left(x_{i}\right)\right)^{2},
$$

where $i=1,2, \ldots, N$, with $N$ denoting the number of computational cross-sections.

For all test cases, rectangular channels of width $b=10 \mathrm{~m}$ with Manning's roughness coefficient $n=0.03 \mathrm{~s} / \mathrm{m}^{1 / 3}$ were assumed. The energy correctional coefficient and gravitational acceleration were assumed as $\alpha=1.1$ and $g=9.81 \mathrm{~m} / \mathrm{s}^{2}$, respectively. To estimate the critical depth, the following formula was used:

$$
H_{C}=\sqrt[3]{\frac{\alpha \cdot Q^{2}}{g \cdot b^{2}}},
$$

where $b$ denotes the channel width. The critical depth level $h_{C}$ for the channel in a given test case was estimated as the sum of the constant $H_{C}$ and the bed level $z(x)$, that is $h_{C}(x)=z(x)+H_{C}$. 


\section{Test Case 1}

Consider a channel of length $L=1000 \mathrm{~m}$. The flow rate in the channel is $Q=15 \mathrm{~m}^{3} / \mathrm{s}$, and the imposed depth in the last cross-section is $H_{L}=1.125 \mathrm{~m}$. The assumed depth function is described by the following equation:

$$
H(x)=\frac{9}{8}+\frac{1}{4} \sin \left(\frac{\pi \cdot x}{\frac{L}{2}}\right) .
$$

The bed level $z(x)$, water stage $h(x)=z(x)+H(x)$, energy line $E(x)$ and critical depth level resulting from Eq. (31) are displayed in Fig. 3.

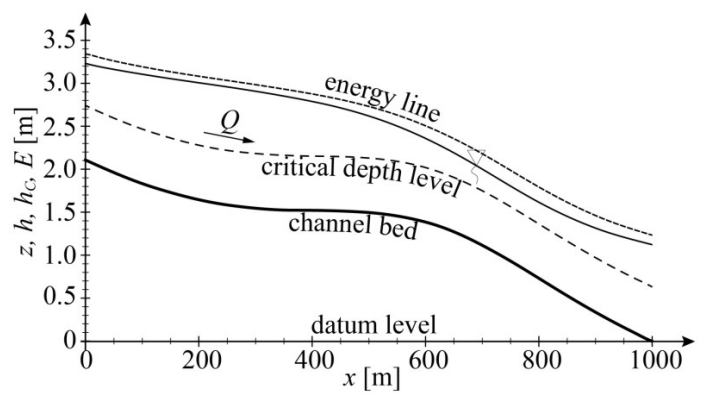

Fig. 3. Plot of the analytic water depth function for test case 1

The ratio of the numerical solution to the analytic solution $H_{i} / H\left(x_{i}\right)$ for computations performed with the step $\Delta x=50 \mathrm{~m}$ is displayed in Fig. 4a. The parabolic and elliptic methods produced much greater errors than other methods. Therefore, to provide a clear view of other solutions, it was necessary to plot them without the parabolic and elliptic methods (Fig. 4b). The horizontal line marked on the vertical axis at 1.0 denotes the analytic depth function.

Errors computed on the basis of Eqs. (29) and (30) are given in Table 1.

Table 1. Test case 1 errors for $\Delta x=50 \mathrm{~m}$

\begin{tabular}{|c|c|c|}
\hline Method & $\delta_{\max }$ & $\sqrt{M S E}$ \\
\hline arithmetic & $2.079 \cdot 10^{-3}$ & $1.215 \cdot 10^{-3}$ \\
hydraulic/RW4 & $2.417 \cdot 10^{-3}$ & $1.347 \cdot 10^{-3}$ \\
geometric & $2.237 \cdot 10^{-3}$ & $1.073 \cdot 10^{-3}$ \\
harmonic & $2.878 \cdot 10^{-3}$ & $1.739 \cdot 10^{-3}$ \\
parabolic & $9.015 \cdot 10^{-3}$ & $6.223 \cdot 10^{-3}$ \\
elliptic & $14.785 \cdot 10^{-3}$ & $10.766 \cdot 10^{-3}$ \\
RW1 & $2.216 \cdot 10^{-3}$ & $1.040 \cdot 10^{-3}$ \\
RW2 & $2.360 \cdot 10^{-3}$ & $1.247 \cdot 10^{-3}$ \\
RW3 & $2.346 \cdot 10^{-3}$ & $1.220 \cdot 10^{-3}$ \\
\hline
\end{tabular}



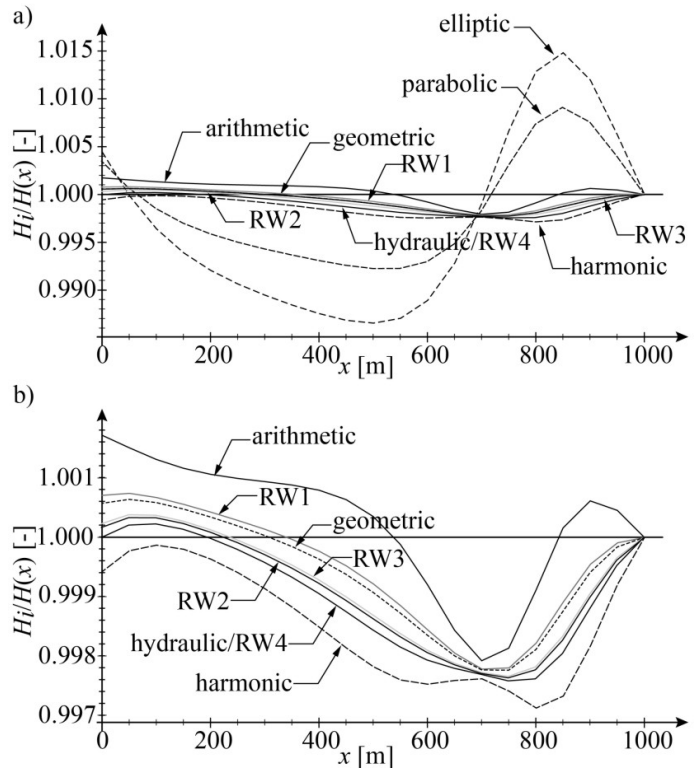

Fig. 4. Test case 1: plots of $H_{i} / H\left(x_{i}\right)$ for $\Delta x=50 \mathrm{~m}$, a) with and b) without the parabolic and elliptic methods

The ratio of the numerical solution to the analytic solution $H_{i} / H\left(x_{i}\right)$ for computations performed with the step $\Delta x=5 \mathrm{~m}$ is displayed in Fig. 5a. The values obtained for the parabolic and elliptic methods are much greater than for others, so it is impossible to distinguish between them. Therefore, another plot (Fig. 5b) was made to provide a clear view.

Errors for computations with the smaller step $\Delta x=5 \mathrm{~m}$ are given in Table 2.

Table 2. Test case 1 errors for $\Delta x=5 \mathrm{~m}$

\begin{tabular}{|c|c|c|}
\hline Method & $\delta_{\max }$ & $\sqrt{M S E}$ \\
\hline arithmetic & $2.041 \cdot 10^{-5}$ & $1.193 \cdot 10^{-5}$ \\
hydraulic/RW4 & $2.412 \cdot 10^{-5}$ & $1.372 \cdot 10^{-5}$ \\
geometric & $2.249 \cdot 10^{-5}$ & $1.088 \cdot 10^{-5}$ \\
harmonic & $2.831 \cdot 10^{-5}$ & $1.771 \cdot 10^{-5}$ \\
parabolic & $92.432 \cdot 10^{-5}$ & $67.384 \cdot 10^{-5}$ \\
elliptic & $157.707 \cdot 10^{-5}$ & $115.626 \cdot 10^{-5}$ \\
RW1 & $2.225 \cdot 10^{-5}$ & $1.051 \cdot 10^{-5}$ \\
RW2 & $2.341 \cdot 10^{-5}$ & $1.266 \cdot 10^{-5}$ \\
RW3 & $2.392 \cdot 10^{-5}$ & $1.242 \cdot 10^{-5}$ \\
\hline
\end{tabular}

Table 3 presents error reduction estimated as the ratio of error for computations with the greater step $(\Delta x=50 \mathrm{~m})$ to error for computations with the smaller step $(\Delta x=5 \mathrm{~m})$. 


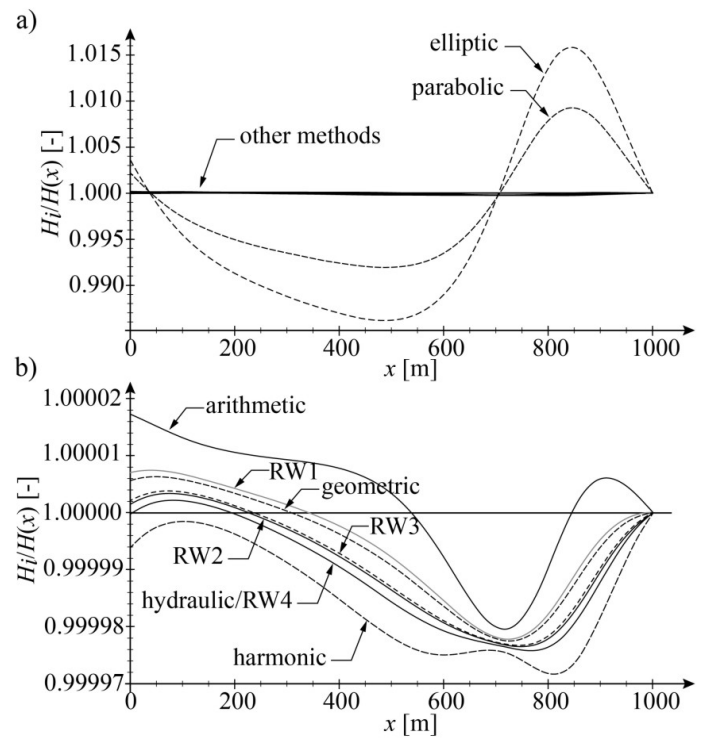

Fig. 5. Test case 1: plots of $H_{i} / H\left(x_{i}\right)$ for $\Delta x=5 \mathrm{~m}$, a) with and b) without the parabolic and elliptic methods

Table 3. Error reduction for test case 1

\begin{tabular}{|c|c|c|}
\hline Method & $\delta_{\max }$ & $\sqrt{M S E}$ \\
\hline arithmetic & 101.86 & 101.84 \\
hydraulic/RW4 & 100.21 & 98.18 \\
geometric & 99.47 & 98.62 \\
harmonic & 101.67 & 98.19 \\
parabolic & 9.75 & 9.24 \\
elliptic & 9.38 & 9.31 \\
RW1 & 99.60 & 98.95 \\
RW2 & 100.81 & 98.50 \\
RW3 & 100.73 & 98.22 \\
\hline
\end{tabular}

The elliptic and parabolic methods performed the worst, giving the greatest error and the least error reduction. In computations with the step size $\Delta x=50 \mathrm{~m}$ the parabolic and elliptic methods gave errors greater than those obtained with the other methods by one order of magnitude. For the numerical experiment carried out with the step size $\Delta x=5 \mathrm{~m}$ the elliptic and parabolic methods gave errors greater than those obtained with the other methods by two orders of magnitude. For both step sizes, the smallest $\delta_{\max }$ errors were obtained for computations with the arithmetic mean. The greatest error reduction, as well, was obtained for computations by arithmetic mean averaging. The least mean square error for both step sizes was obtained by averaging method denoted as RW1. 


\section{Test Case 2}

In this test case, computations were performed for a channel of length $L=1000 \mathrm{~m}$, and the flow rate $Q=20 \mathrm{~m}^{3} / \mathrm{s}$. The imposed depth in the last cross-section is $H_{L}=0.8$ $\mathrm{m}$, which is greater than the critical depth. The assumed depth function, representing the drawdown profile, is described by the following equation:

$$
H(x)=\frac{37}{1000} \sqrt{L-x}+\frac{8}{10} .
$$

The plot of the bed level, water stage, energy line and critical depth level resulting from Eq. (32) is displayed in Fig. 6.

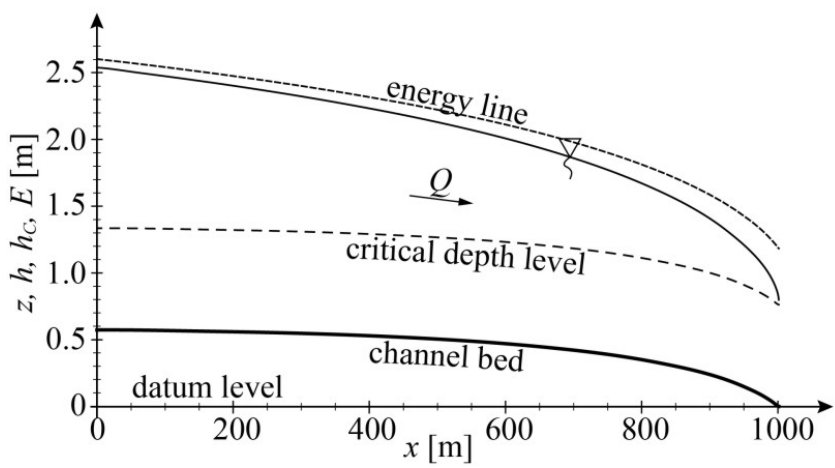

Fig. 6. Plot of the analytic water depth function for test case 2

First, computations were performed with the spatial step $\Delta x=50 \mathrm{~m}$. The plot of the ratio $H_{i} / H\left(x_{i}\right)$ is given in Fig. 7. The errors obtained are given in Table 4.

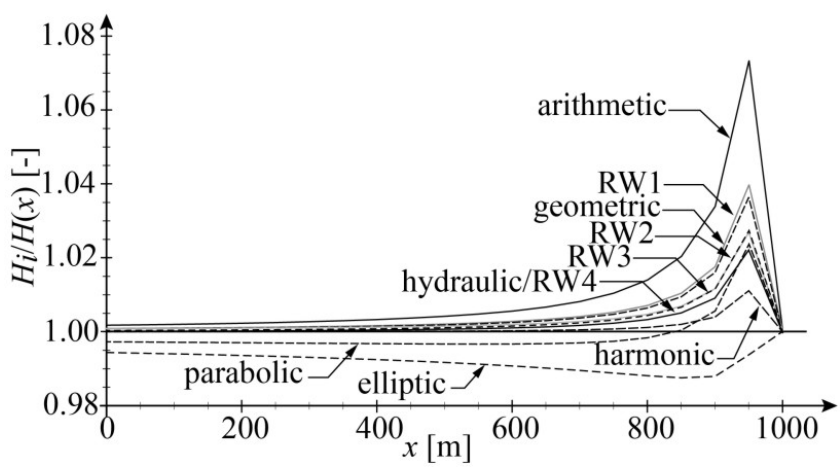

Fig. 7. Test case 1: plots of $H_{i} / H\left(x_{i}\right)$ for $\Delta x=50 \mathrm{~m}$

For $\Delta x=5 \mathrm{~m}$, the plot of the ratio $H_{i} / H\left(x_{i}\right)$ is given in Fig. 8, the estimated errors are given in Table 5 and the error reduction is presented in Table 6. 
Table 4. Test case 2 errors for $\Delta x=50 \mathrm{~m}$

\begin{tabular}{|c|c|c|}
\hline Method & $\delta_{\max }$ & $\sqrt{M S E}$ \\
\hline arithmetic & $7.321 \cdot 10^{-2}$ & $2.116 \cdot 10^{-2}$ \\
hydraulic/RW4 & $2.231 \cdot 10^{-2}$ & $0.613 \cdot 10^{-2}$ \\
geometric & $3.627 \cdot 10^{-2}$ & $1.022 \cdot 10^{-2}$ \\
harmonic & $1.110 \cdot 10^{-2}$ & $0.286 \cdot 10^{-2}$ \\
parabolic & $2.380 \cdot 10^{-2}$ & $0.722 \cdot 10^{-2}$ \\
elliptic & $1.250 \cdot 10^{-2}$ & $1.289 \cdot 10^{-2}$ \\
RW1 & $3.967 \cdot 10^{-2}$ & $1.123 \cdot 10^{-2}$ \\
RW2 & $2.650 \cdot 10^{-2}$ & $0.736 \cdot 10^{-2}$ \\
RW3 & $2.749 \cdot 10^{-2}$ & $0.765 \cdot 10^{-2}$ \\
\hline
\end{tabular}

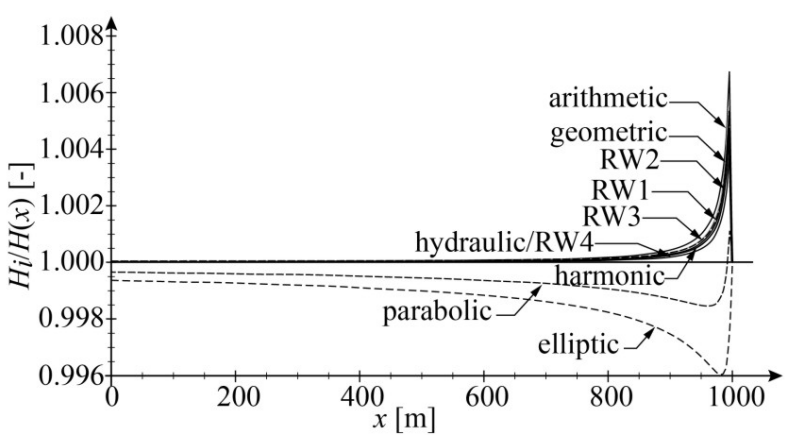

Fig. 8. Test case 2: plots of $H_{i} / H\left(x_{i}\right)$ for $\Delta x=5 \mathrm{~m}$

Table 5. Test case 2 errors for $\Delta x=5 \mathrm{~m}$

\begin{tabular}{|c|c|c|}
\hline Method & $\delta_{\max }$ & $\sqrt{M S E}$ \\
\hline arithmetic & $6.736 \cdot 10^{-3}$ & $0.666 \cdot 10^{-3}$ \\
hydraulic/RW4 & $4.644 \cdot 10^{-3}$ & $0.441 \cdot 10^{-3}$ \\
geometric & $5.323 \cdot 10^{-3}$ & $0.514 \cdot 10^{-3}$ \\
harmonic & $3.987 \cdot 10^{-3}$ & $0.370 \cdot 10^{-3}$ \\
parabolic & $1.094 \cdot 10^{-3}$ & $1.035 \cdot 10^{-3}$ \\
elliptic & $3.965 \cdot 10^{-3}$ & $1.986 \cdot 10^{-3}$ \\
RW1 & $5.466 \cdot 10^{-3}$ & $0.530 \cdot 10^{-3}$ \\
RW2 & $4.865 \cdot 10^{-3}$ & $0.465 \cdot 10^{-3}$ \\
RW3 & $4.911 \cdot 10^{-3}$ & $0.470 \cdot 10^{-3}$ \\
\hline
\end{tabular}

In this test, the greatest maximum relative error $\delta_{\max }$ was obtained for the method using the arithmetic mean for energy slope averaging with both step sizes. For computations with the step size of $50 \mathrm{~m}$, the least $\delta_{\max }$ was obtained for energy slope averaging with the harmonic mean. In computations with the smaller step size, the least $\delta_{\max }$ was obtained for the parabolic method, which was designed for such flow profiles. The least mean square error was obtained for both step sizes when harmonic mean averaging was used. 
Table 6. Error reduction for test case 2

\begin{tabular}{|c|r|r|}
\hline Method & \multicolumn{1}{|c|}{$\delta_{\max }$} & $\sqrt{M S E}$ \\
\hline arithmetic & 10.87 & 31.77 \\
hydraulic/RW4 & 4.81 & 13.91 \\
geometric & 6.82 & 19.88 \\
harmonic & 2.78 & 7.73 \\
parabolic & 21.76 & 6.98 \\
elliptic & 3.15 & 6.49 \\
RW1 & 7.26 & 21.20 \\
RW2 & 5.45 & 15.84 \\
RW3 & 5.60 & 16.29 \\
\hline
\end{tabular}

\section{Test Case 3}

This test case represents a typical backwater water profile. To obtain the analytic depth function for this case, a synthetic example of a backwater curve was formulated and solved numerically. The numerical solution obtained was approximated with a second-degree polynomial by the least squares method. The depth function obtained is

$$
H(x)=5.716 \cdot 10^{-7} x^{2}-4.392 \cdot 10^{-5} x+0.44,
$$

and it is displayed in Fig. 9. The given channel has length $L=1000 \mathrm{~m}$ and the flow rate $Q=2.5 \mathrm{~m}^{3} / \mathrm{s}$. The imposed depth in the last cross-section is $H_{L}=0.9677 \mathrm{~m}$.

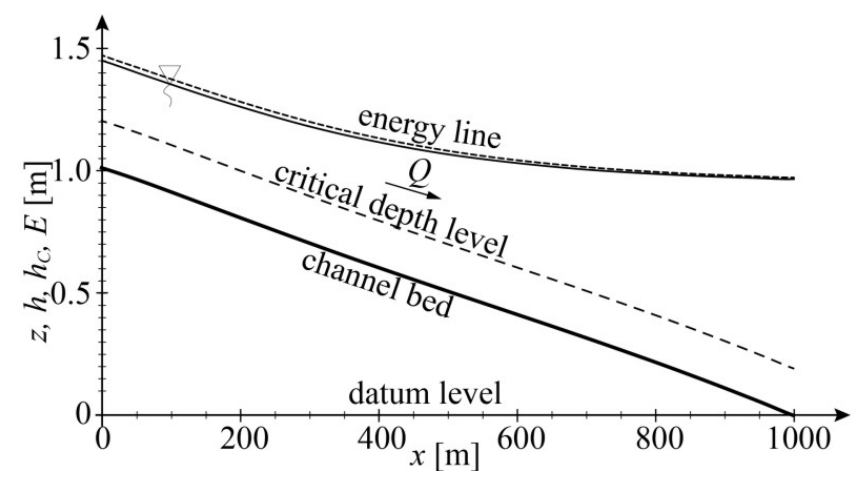

Fig. 9. lot of the analytic water depth function for test case 3

Errors for each computation method with the step size $\Delta x=50 \mathrm{~m}$ are presented in Table 7. All relative water depths $H_{i} / H(x)$ are displayed in Fig. 10a, whereas Fig. $10 \mathrm{~b}$ shows relative depths with the elliptic and parabolic methods neglected. 
Table 7. Test case 3 errors for $\Delta x=50 \mathrm{~m}$

\begin{tabular}{|c|c|c|}
\hline Method & $\delta_{\max }$ & $\sqrt{M S E}$ \\
\hline arithmetic & $4.082 \cdot 10^{-4}$ & $1.120 \cdot 10^{-4}$ \\
hydraulic/RW4 & $7.753 \cdot 10^{-4}$ & $2.518 \cdot 10^{-4}$ \\
geometric & $5.445 \cdot 10^{-4}$ & $1.576 \cdot 10^{-4}$ \\
harmonic & $10.867 \cdot 10^{-4}$ & $3.515 \cdot 10^{-4}$ \\
parabolic & $61.326 \cdot 10^{-4}$ & $19.171 \cdot 10^{-4}$ \\
elliptic & $102.433 \cdot 10^{-4}$ & $32.129 \cdot 10^{-4}$ \\
RW1 & $5.307 \cdot 10^{-4}$ & $1.416 \cdot 10^{-4}$ \\
RW2 & $6.735 \cdot 10^{-4}$ & $2.182 \cdot 10^{-4}$ \\
RW3 & $6.618 \cdot 10^{-4}$ & $2.132 \cdot 10^{-4}$ \\
\hline
\end{tabular}
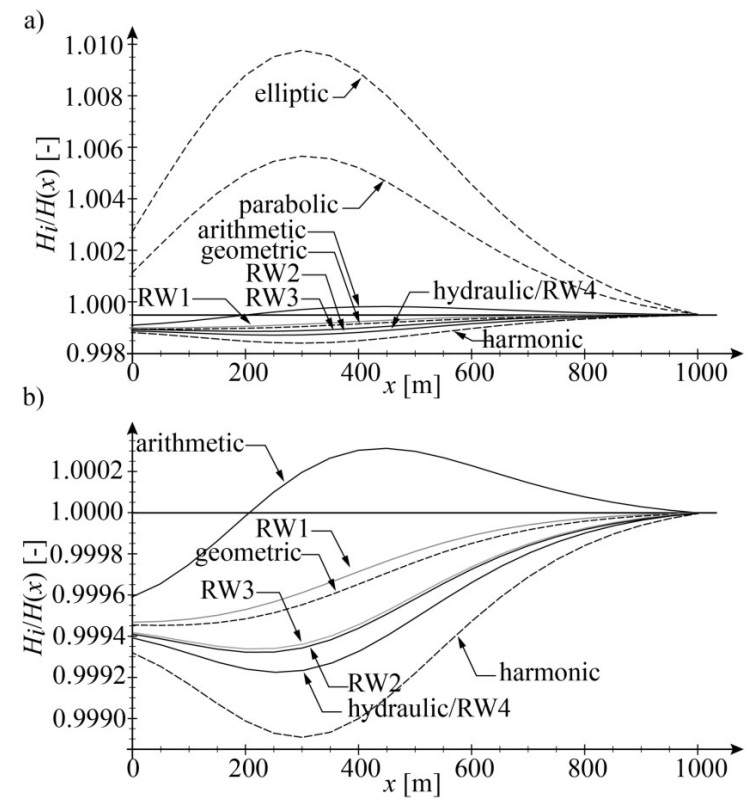

Fig. 10. Plots of $H_{i} / H\left(x_{i}\right)$ for $\Delta x=50 \mathrm{~m}$

Table 8. Test case 3 errors for $\Delta x=5 \mathrm{~m}$

\begin{tabular}{|c|c|c|}
\hline Method & $\delta_{\max }$ & $\sqrt{M S E}$ \\
\hline arithmetic & $4.067 \cdot 10^{-6}$ & $1.111 \cdot 10^{-6}$ \\
hydraulic/RW4 & $7.736 \cdot 10^{-6}$ & $2.540 \cdot 10^{-6}$ \\
geometric & $5.441 \cdot 10^{-6}$ & $1.570 \cdot 10^{-6}$ \\
harmonic & $10.833 \cdot 10^{-6}$ & $3.561 \cdot 10^{-6}$ \\
parabolic & $604.638 \cdot 10^{-6}$ & $191.754 \cdot 10^{-6}$ \\
elliptic & $1032.750 \cdot 10^{-6}$ & $327.639 \cdot 10^{-6}$ \\
RW1 & $5.303 \cdot 10^{-6}$ & $1.404 \cdot 10^{-6}$ \\
RW2 & $6.742 \cdot 10^{-6}$ & $2.195 \cdot 10^{-6}$ \\
RW3 & $6.616 \cdot 10^{-6}$ & $2.143 \cdot 10^{-6}$ \\
\hline
\end{tabular}


Errors for each computation method with the step size $\Delta x=5 \mathrm{~m}$ are presented in Table 8, whereas the plot of relative water depths is displayed in Fig. 11a. Another plot (Fig. 11b) was made, neglecting the parabolic and elliptic methods, to make the other methods distinguishable. The reduction of errors is presented in Table 9.

Table 9. Error reduction for test case 3

\begin{tabular}{|c|c|c|}
\hline Method & $\delta_{\max }$ & $\sqrt{M S E}$ \\
\hline arithmetic & 100.37 & 100.81 \\
hydraulic/RW4 & 100.22 & 99.13 \\
geometric & 100.07 & 100.34 \\
harmonic & 100.31 & 98.72 \\
parabolic & 10.14 & 100.00 \\
elliptic & 9.92 & 9.81 \\
RW1 & 100.08 & 100.85 \\
RW2 & 99.90 & 99.39 \\
RW3 & 100.03 & 99.481 \\
\hline
\end{tabular}

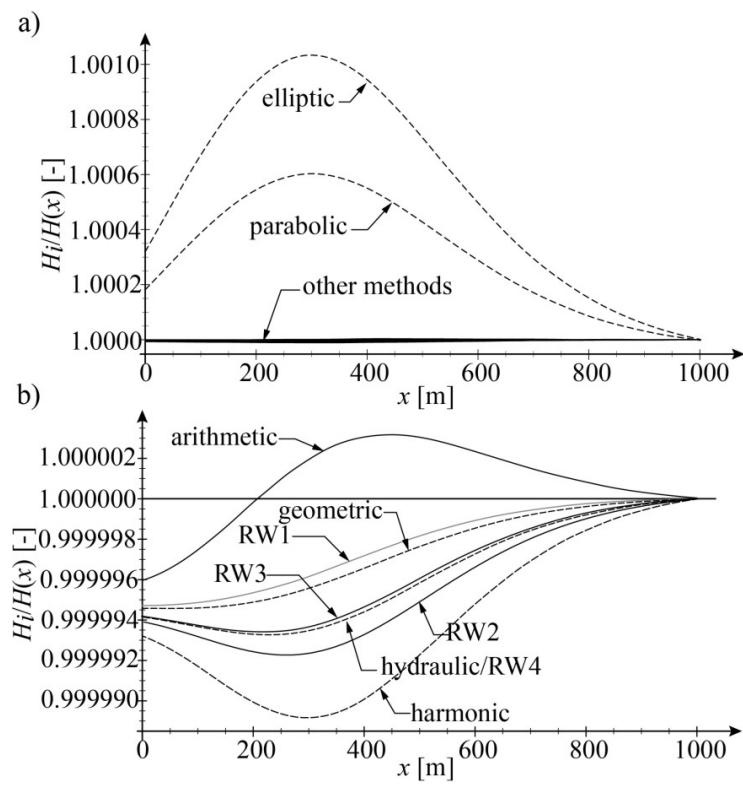

Fig. 11. Plots of $H_{i} / H(x)$ for $\Delta x=5 \mathrm{~m}$

The parabolic and elliptic methods were developed for drawdown profiles. Therefore, when applied to computations of a backwater profile, they produced the greatest relative maximum and mean square errors, for both step sizes. The least maximum relative and mean square errors were obtained for the method using arithmetic mean averaging for both step sizes. 


\section{Test Case 4}

This test case represents rapid flow in a rectangular channel of length $L=15 \mathrm{~m}$. The flow rate is $Q=22 \mathrm{~m}^{3} / \mathrm{s}$, and the imposed depth in the first cross-section is $H_{L}=$ $0.7341 \mathrm{~m}$, which is less than the critical depth $H_{C}=0.816 \mathrm{~m}$. The assumed depth function is described by the following equation:

$$
H(x)=-0.1 \sqrt{x}+0.9 H_{C} .
$$

The channel bed, critical depth level, water profile $H(x)$ and energy line for this test case are presented in Fig. 12.

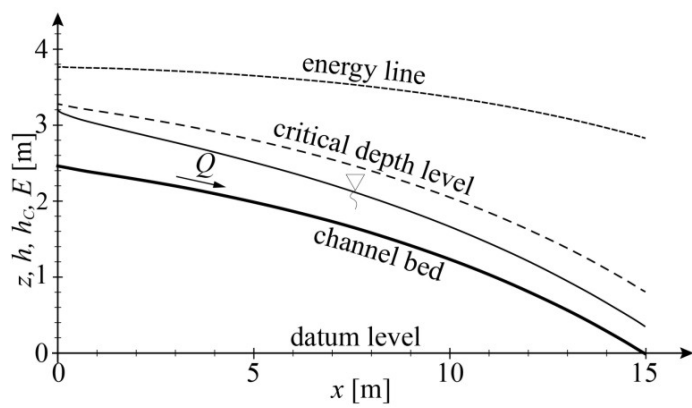

Fig. 12. Plots of the analytic water depth function for test case 4

Computations for this test case were performed with step size values of $3 \mathrm{~m}$ and $0.3 \mathrm{~m}$. The errors for the former $(\Delta x=3 \mathrm{~m})$ are presented in Table 10, whereas the plot of relative depths is displayed in Fig. 13.

Table 10. Test case 4 errors for $\Delta x=3 \mathrm{~m}$

\begin{tabular}{|c|c|c|}
\hline Method & $\delta_{\max }$ & $\sqrt{M S E}$ \\
\hline arithmetic & $3.061 \cdot 10^{-3}$ & $0.765 \cdot 10^{-3}$ \\
hydraulic/RW4 & $10.497 \cdot 10^{-3}$ & $2.992 \cdot 10^{-3}$ \\
geometric & $8.014 \cdot 10^{-3}$ & $2.197 \cdot 10^{-3}$ \\
harmonic & $12.821 \cdot 10^{-3}$ & $3.750 \cdot 10^{-3}$ \\
parabolic & $11.261 \cdot 10^{-3}$ & $3.260 \cdot 10^{-3}$ \\
elliptic & $18.103 \cdot 10^{-3}$ & $5.612 \cdot 10^{-3}$ \\
RW1 & $7.541 \cdot 10^{-3}$ & $2.049 \cdot 10^{-3}$ \\
RW2 & $9.653 \cdot 10^{-3}$ & $2.717 \cdot 10^{-3}$ \\
RW3 & $9.521 \cdot 10^{-3}$ & $2.677 \cdot 10^{-3}$ \\
\hline
\end{tabular}

The errors produced by different methods for the smaller step $(\Delta x=0.3 \mathrm{~m})$ are presented in Table 11, whereas relative depths are depicted in Fig. 14. The error reduction is presented in Table 12 .

The smallest mean square error was produced by the arithmetic method for both step sizes. For computations with the step size $\Delta x=3 \mathrm{~m}$, the least $\delta_{\max }$ error was obtained by the arithmetic averaging of the energy slope, whereas the greatest $\delta_{\max }$ was 


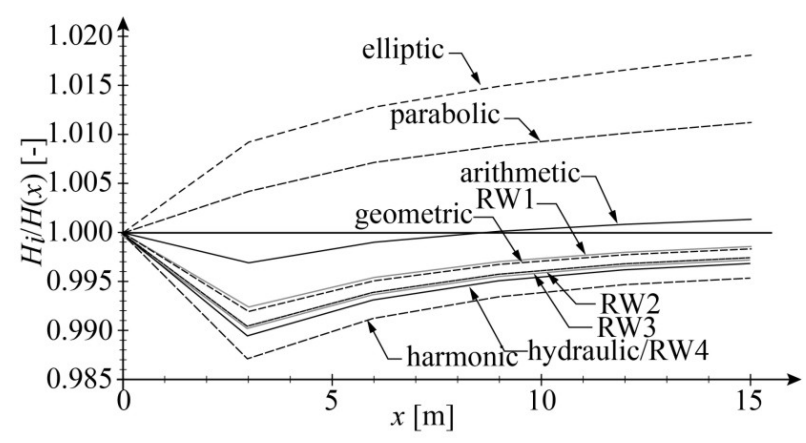

Fig. 13. Plots of $H_{i} / H(x)$ for $\Delta x=3 \mathrm{~m}$

Table 11. Test case 4 errors for $\Delta x=0.3 \mathrm{~m}$

\begin{tabular}{|c|c|c|}
\hline Method & $\delta_{\max }$ & $\sqrt{M S E}$ \\
\hline arithmetic & $3.581 \cdot 10^{-3}$ & $0.368 \cdot 10^{-3}$ \\
hydraulic/RW4 & $3.478 \cdot 10^{-3}$ & $0.369 \cdot 10^{-3}$ \\
geometric & $3.512 \cdot 10^{-3}$ & $0.368 \cdot 10^{-3}$ \\
harmonic & $3.444 \cdot 10^{-3}$ & $0.370 \cdot 10^{-3}$ \\
parabolic & $3.972 \cdot 10^{-3}$ & $0.511 \cdot 10^{-3}$ \\
elliptic & $4.248 \cdot 10^{-3}$ & $0.723 \cdot 10^{-3}$ \\
RW1 & $3.519 \cdot 10^{-3}$ & $0.368 \cdot 10^{-3}$ \\
RW2 & $3.490 \cdot 10^{-3}$ & $0.369 \cdot 10^{-3}$ \\
RW3 & $3.492 \cdot 10^{-3}$ & $0.369 \cdot 10^{-3}$ \\
\hline
\end{tabular}

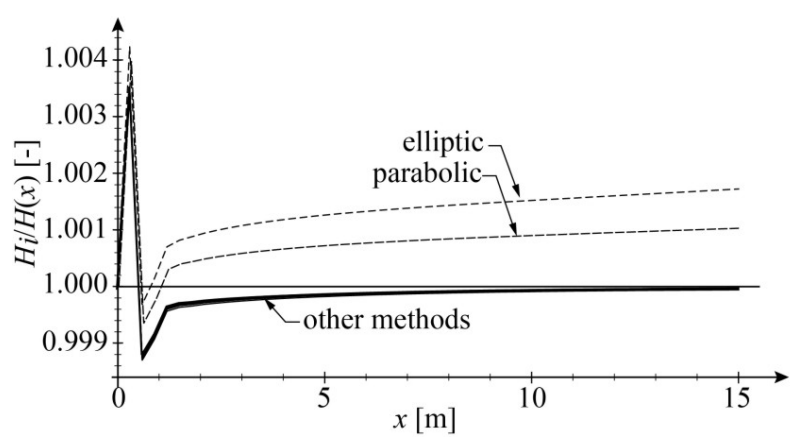

Fig. 14. Plots of $H_{i} / H(x)$ for $\Delta x=0.3 \mathrm{~m}$

obtained for the elliptic method. For the smaller step size, the least $\delta_{\max }$ was obtained by harmonic mean averaging. What is interesting, the arithmetic method gave the maximum absolute and relative error reduction smaller than one. This means that the maximum error actually increased when the step size was reduced. This phenomenon is explained by the placement of the computational cross-sections. Mean square error of this method was reduced by half. 
Table 12. Error reduction for test case 4

\begin{tabular}{|c|c|c|}
\hline Method & $\delta_{\max }$ & $\sqrt{M S E}$ \\
\hline arithmetic & 0.86 & 2.08 \\
hydraulic/RW4 & 3.02 & 8.11 \\
geometric & 2.28 & 5.96 \\
harmonic & 3.72 & 10.14 \\
parabolic & 2.84 & 6.38 \\
elliptic & 4.26 & 7.76 \\
RW1 & 2.14 & 5.57 \\
RW2 & 2.77 & 7.37 \\
RW3 & 2.73 & 7.26 \\
\hline
\end{tabular}

\section{Conclusions}

Numerical experiments showed that all methods examined can be successfully applied in engineering practice, as all numerical solutions turned out to be very close to the analytical ones. Moreover, no stability or convergence problems were observed.

The indicators of the quality of the methods examined were relative maximum and mean square errors. In the test cases considered, the errors were relatively small from the practical point of view.

For each water profile, the methods suggested in the literature were confronted with computation outcomes. The first test case represented a typical water flow profile found in open-channel hydraulics, which contains channel reaches with different water profiles. In this case, the arithmetic mean RW1 averaging method turned out to be the best.

For a drawdown profile (test case 2), the method suggested by French (1979) is geometric mean averaging. However, harmonic mean averaging turned out to be the best, giving the least mean square error, regardless of the step size. Harmonic mean averaging is the method suggested for such profiles by the US Army Corps of Engineers (2010). The standard step method (arithmetic averaging) turned out to be the worst in this case, producing the greatest errors.

For the backwater curve example (test case 3), the averaging method suggested by French is hydraulic averaging. However, the best performance was obtained with the arithmetic averaging method, recommended by the US Army Corps of Engineers (2010).

For cases like the spillway example (test case 4), French (1979) suggests hydraulic averaging, but arithmetic mean averaging, recommended by the US Army Corps of Engineers (2010), performed better.

The hydraulic averaging method (default HEC-RAS energy slope averaging method), did not prove generally better than any other. It gave errors similar to other methods. However, its errors were greater than those for the methods suggested for specific types of water flow profiles. In fact, in most cases the standard step method gave smaller error. 
From the numerical point of view, it seems reasonable to use different methods for different profiles. In reality, however, the biggest problem and the source of errors is roughness estimation for cross-sections of natural channels. Therefore, numerical error seems of less importance than it was assumed.

The result of energy slope averaging based on the parameters of neighbouring cross-sections is closely related to the choice of method for the numerical solution of the IVP for an ODE. This problem requires further analyses because, apart from the accuracy of the method, also other numerical properties, such as stability, convergence, and the ability to conserve physical quantities, are important.

\section{References}

Ascher U. M., Petzold L. R. (1998) Computer methods for Ordinary Differential Equations and Difference-Algebraic Equations, SIAM, Philadelphia.

Artichowicz W., Szymkiewicz R. (2014) Computational issues of solving the 1D steady gradually varied flow equation, J. Hydrol. Hydromech., 62 (3), 226-233. DOI: 10.2478/johh-2014-0031.

Chanson H. (2004) The hydraulics of open channel flow: an introduction. Second Edition. Elsevier.

Chadderton R. A., Miller A. C. (1980) Friction models for M2 profiles, JAWRA Journal of the American Water Resources Association, 16 (2), 235-242, DOI: 10.1111/j.1752-1688.1980.tb02384.x.

Cunge J. A., Holly F. M., Verwey A. (1979) Practical aspects of computational river hydraulics, Pitman advanced publishing program, Boston, London, Melbourne.

French R. H. (1985) Open Channel Hydraulics, McGraw-Hill, New York.

Hairer E., Lubich C., Wanner G. (2005) Geometric Numerical Integration. Structure-Preserving Algorithms for Ordinary Differential Equations. Second Edition. Springer, Berlin, Heidelberg, New York.

Kincaid D., Cheney W. (2006) Numerical Analysis: Mathematics of Scientific Computing (Polish edition). Wydawnictwa Naukowo-Techniczne, Warszawa.

Laurenson E. M. (1986) Friction Slope Averaging in Backwater Calculations, J. Hydraul. Eng., 112 (12), 1151-1163.

MacDonald I., Baines M. J., Nichols N. K., Samuels P. G. (1997) Analytic Benchmark Solutions for Open-Channel Flows, J. Hydraul. Eng., 123 (11), 1041-1045.

Szymkiewicz R. (2010) Numerical modeling in open channel hydraulics, Springer.

US Army Corps of Engineers (2010) HEC-RAS hydraulic reference. 